\title{
Contact-line deposits from multiple evaporating droplets
}

\author{
Alexander W. Wray $\odot^{*}$ \\ Department of Mathematics and Statistics, University of Strathclyde, Livingstone Tower, \\ 26 Richmond Street, Glasgow, G1 1XH, United Kingdom \\ Patrick S. Wray ${ }^{\dagger}$ \\ Drug Product Science and Technology, Bristol-Myers Squibb, Reeds Lane, Moreton, Wirral, CH46 1QW, \\ United Kingdom \\ Brian R. Duffy ${ }^{\ddagger}$ and Stephen K. Wilson $\oplus^{\S}$ \\ Department of Mathematics and Statistics, University of Strathclyde, Livingstone Tower, \\ 26 Richmond Street, Glasgow, G1 1XH, United Kingdom
}

(Received 8 January 2021; accepted 25 June 2021; published 22 July 2021)

\begin{abstract}
Building on the recent theoretical work of Wray et al. [J. Fluid Mech. 884, A45 (2020)] concerning the competitive diffusion-limited evaporation of multiple thin sessile droplets in proximity to each other, we obtain theoretical predictions for the spatially nonuniform densities of the contact-line deposits (often referred to as "coffee stains" or "ring stains") left on the substrate after such droplets containing suspended solid particles have completely evaporated. Neighboring droplets interact via their vapour fields, which results in a spatially nonuniform "shielding" effect. We give predictions for the deposits from a pair of identical droplets, which show that the deposit is reduced the most where the droplets are closest together, and demonstrate excellent quantitative agreement with experimental results of Pradhan and Panigrahi [Colloids Surf. A Physicochem. Eng. Asp. 482, 562 (2015)]. We also give corresponding predictions for a triplet of identical droplets arranged in an equilateral triangle, which show that the effect of shielding on the deposit is more subtle in this case.
\end{abstract}

DOI: 10.1103/PhysRevFluids.6.073604

\section{INTRODUCTION}

The evaporation of sessile droplets has been the subject of extensive experimental, numerical and analytical investigation in recent years (see, for example, Refs. [1-5] and the references therein), partly motivated by the wide range of everyday and industrial situations, such as protein crystallography [6], surface patterning [7], ink-jet printing, including that of OLED displays [8], and agrochemical spraying of plants [9], in which it occurs.

\footnotetext{
*alexander.wray@strath.ac.uk

†patrick.wray@bms.com

†.r.duffy@strath.ac.uk

${ }^{\S}$ Author for correspondence: s.k.wilson@strath.ac.uk
}

Published by the American Physical Society under the terms of the Creative Commons Attribution 4.0 International license. Further distribution of this work must maintain attribution to the author(s) and the published article's title, journal citation, and DOI. 
Particular attention has been paid to the so-called "coffee-stain" or "ring-stain" effect: when a droplet of coffee (or indeed a droplet of any fluid containing suspended solid particles) with a pinned (i.e., a fixed) contact line evaporates it tends to deposit the majority of the particles close to the location of its contact line, even if the particles were initially distributed uniformly throughout the bulk of the droplet. The explanation of this phenomenon, as first given by Deegan et al. [10], is that as the droplet evaporates its free surface adjusts quasistatically under the effect of capillarity, inducing a flow within the droplet that advects the particles suspended within it toward its contact line, resulting in a characteristic ring-like contact-line deposit on the substrate after the droplet has completely evaporated. Since the seminal work by Deegan et al. [10], many aspects of this phenomenon have been investigated in considerable detail (see, for example, Refs. [11-25] and the reviews in Refs. [2,26,27]). Note that although Deegan et al. [10] considered the most commonly studied situation of diffusion-limited evaporation into a quiescent atmosphere with a uniform farfield concentration of vapour, which has a large (theoretically singular) evaporative flux close to the contact line, the effect is quite robust, and even, for example, a spatially uniform evaporative flux will lead to advection of particles toward the contact line (see, for example, Refs. [12,14,23]).

The vast majority of the previous work on deposition from evaporating sessile droplets has, for obvious reasons, focused on axisymmetric deposits from axisymmetric droplets. There has, however, been some work on nonaxisymmetric deposits from nonaxisymmetric droplets (see, for example, Refs. [9,10,28-32]). In particular, Du and Deegan [29] examined a two-dimensional droplet on an inclined substrate numerically, and found that, depending on the initial volume of the droplet and the angle of inclination of the substrate, the larger deposit can occur at either the upper or the lower contact line, while Sáenz et al. [31] investigated a variety of nonaxisymmetric droplets both experimentally and numerically, and found that larger deposits occur where the contact line has the largest curvature (e.g., near the tips of a droplet with a triangular contact line). However, their theoretical modeling of the density of the deposit was essentially phenomenological.

Nonaxisymmetric deposits also occur as a result of the nonaxisymmetric evaporation of multiple droplets in proximity to each other, a situation that occurs much more commonly in practice than single droplets in isolation [8]. Specifically, neighboring droplets undergoing diffusion-limited evaporation interact via their vapour fields, which results in a spatially nonuniform "shielding" effect that reduces the evaporation rate. While there have been some analytical studies of mathematically analogous situations concerning clusters of micro-contacts and nanobubbles (see, for example, Refs. $[33,34]$ ), analytical work on the evaporation of multiple droplets is rather limited. To a large extent this is explained by the inherent difficulty of analyzing such situations, and while the evaporation of multiple droplets in various configurations has been the subject of growing recent interest (see, for example, Refs. [12,35-48]), the previous studies have been predominantly numerical or experimental. Two notable exceptions are the recent work of Wray et al. [48], who, building on the earlier work of Fabrikant [49] concerning a model for diffusion through a porous membrane, analyzed the spatially nonuniform shielding that occurs in arbitrary configurations of thin droplets with circular contact lines, and that of Schofield et al. [47], who used conformalmapping techniques to analyze the analogous spatially nonuniform shielding that occurs in the closely related two-dimensional situation of a pair of evaporating ridges. In particular, Wray et al. [48] gave explicit formulas for the evaporative flux of arbitrary configurations of droplets that were found to be remarkably accurate up to and including the limit of touching droplets, and led to theoretical predictions for the evolution of an arrangement of seven droplets that were found to be in excellent agreement with experimental results of Khilifi et al. [45].

In the present contribution we build on the work of Wray et al. [48] in order to analyze the spatially nonuniform densities of the deposits left on the substrate by the diffusion-limited evaporation of multiple thin droplets with pinned circular contact lines in proximity to each other. Specifically, in Secs. II and III we formulate and solve the evaporation, hydrodynamic, and particle-transport problems. In Sec. IV we give theoretical predictions for the densities of the deposits from a pair of identical droplets, and demonstrate excellent quantitative agreement with experimental results of Pradhan and Panigrahi [39]. In Sec. V we also give corresponding predictions for a triplet of 


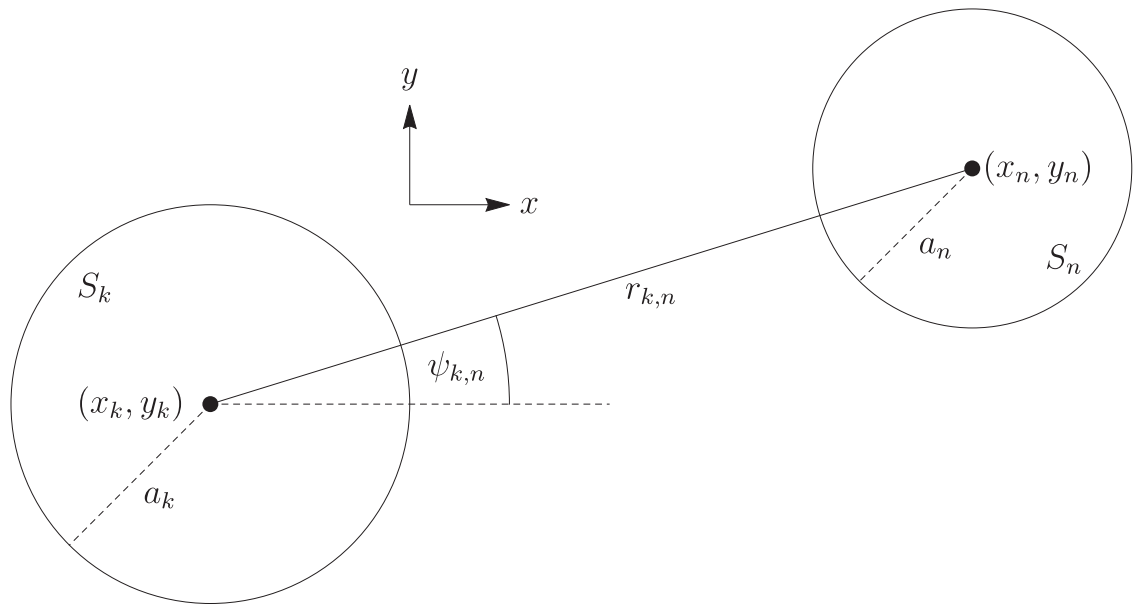

(a)

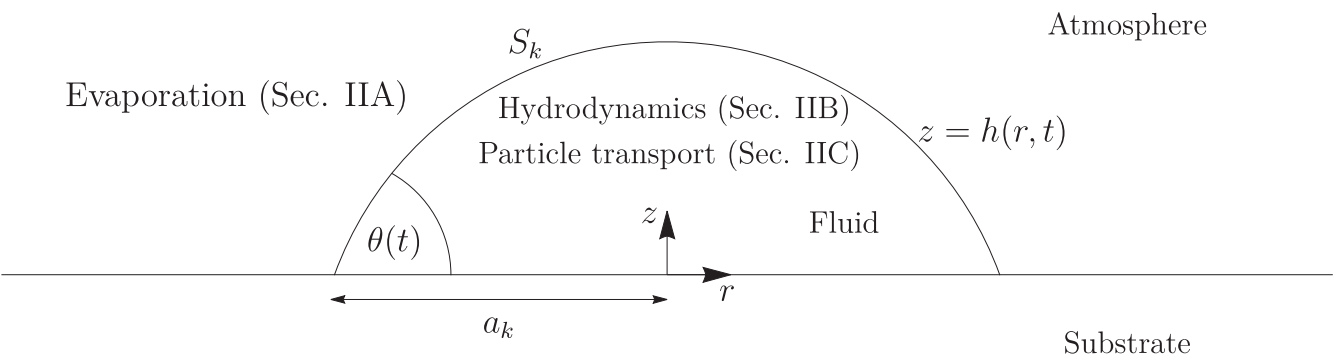

(b)

FIG. 1. (a) Geometry of the $k$ th and $n$th droplets on the substrate $z=0$. (b) A slice through the $k$ th droplet showing the geometry of the droplet and the problems to be solved in the different regions.

identical droplets arranged in an equilateral triangle. Finally, we summarize our conclusions in Sec. VI.

The present analysis is for the most commonly studied case of small droplets, in which capillary effects dominate over gravitational effects, corresponding to the limit of small Bond number. In the Appendix we describe the corresponding analysis for the less commonly studied case of large droplets, corresponding to the limit of large Bond number, in which even greater analytical progress is possible.

\section{PROBLEM FORMULATION}

Consider $N(N=1,2,3, \ldots)$ thin axisymmetric sessile droplets with pinned circular contact lines with constant radii $a_{k}$ and fixed centers at $\left(x_{k}, y_{k}\right)$ for $k=1,2, \ldots, N$ on a planar solid substrate $z=0$, as shown in Fig. 1(a). The droplets undergo quasistatic diffusion-limited evaporation, which, as described in Sec. I, induces flows within the droplets that advect the solid particles suspended within them toward their contact lines. The goal of the present work is to determine the spatially nonuniform densities of the deposits left on the substrate after the droplets have completely evaporated. Three coupled problems must therefore be solved: the evaporation problem for the concentration of vapour in the atmosphere (which determines the rates of evaporation of the droplets), the hydrodynamic problem for the fluid flow that is induced in each droplet, and 
the advection problem for the motion of the particles suspended within each droplet, as shown in Fig. 1(b). We now discuss each of these problems in turn.

\section{A. The evaporation problem}

According to the diffusion-limited model, the quasistatic concentration of vapour in the atmosphere, denoted by $c^{(\mathrm{v})}=c^{(\mathrm{v})}(r, \phi, z)$, satisfies Laplace's equation $\nabla^{2} c^{(\mathrm{v})}=0$ subject to conditions of complete saturation at the free surfaces of the droplets and of no flux of vapour through the unwetted part of the substrate. Following Wray et al. [48], we scale and nondimensionalize variables appropriately for the atmosphere according to

$$
\begin{gathered}
x=a_{\mathrm{ref}} x^{*}, \quad y=a_{\mathrm{ref}} y^{*}, \quad z=a_{\mathrm{ref}} z^{*}, \quad r=a_{\mathrm{ref}} r^{*}, \\
c^{(\mathrm{v})}=c_{\infty}^{(\mathrm{v})}+\left(c_{\mathrm{sat}}^{(\mathrm{v})}-c_{\infty}^{(\mathrm{v})}\right) c^{(\mathrm{v}) *}, \quad J_{k}=\frac{D^{(\mathrm{v})}\left(c_{\mathrm{sat}}^{(\mathrm{v})}-c_{\infty}^{(\mathrm{v})}\right)}{a_{\mathrm{ref}}} J_{k}^{*}, \quad F_{k}=D^{(\mathrm{v})}\left(c_{\mathrm{sat}}^{(\mathrm{v})}-c_{\infty}^{(\mathrm{v})}\right) a_{\mathrm{ref}} F_{k}^{*},
\end{gathered}
$$

where $x$ and $y$ are Cartesian coordinates in the plane $z=0, r, \phi$, and $z$ are local polar coordinates with their origin at the center of the $k$ th droplet, $a_{\text {ref }}$ is a characteristic radius of the contact lines of the droplets, $D^{(\mathrm{v})}$ is the constant diffusion coefficient of vapour in the atmosphere, $c_{\mathrm{sat}}^{(\mathrm{v})}$ and $c_{\infty}^{(\mathrm{v})}$ are the constant saturation concentration and far-field concentration of vapour, and $J_{k}=J_{k}(r, \phi)$ and $F_{k}$ are the local evaporative flux and the integral evaporative flux from the $k$ th droplet, respectively, which are related by

$$
F_{k}=\iint_{S_{k}} J_{k} d S
$$

where $S_{k}$ denotes the free surface of the $k$ th droplet. Since the contact lines of the droplets are pinned, $J_{k}$ and $F_{k}$ are independent of time except for discontinuous jumps when any droplet completely evaporates; in particular, $J_{k}$ and $F_{k}$ jump instantaneously to zero when the $k$ th droplet completely evaporates.

For clarity, we immediately drop the star superscripts on nondimensional quantities, and so the boundary conditions on $c^{(\mathrm{v})}$ become $c^{(\mathrm{v})}=1$ on $z=h_{k}$ for $k=1,2, \ldots, N, \partial c^{(\mathrm{v})} / \partial z=0$ on the unwetted part of the substrate $z=0$, and the far-field condition $c^{(\mathrm{v})} \rightarrow 0$ as $r^{2}+z^{2} \rightarrow \infty$.

As Wray et al. [48] described, the earlier work of Fabrikant [49] on diffusion through a porous membrane, when interpreted in terms of the evaporation of multiple thin sessile droplets, shows that the integral evaporative flux $F_{k}$ is given, to a high degree of accuracy, by the solution of the linear system

$$
F_{k}=4 a_{k}-\frac{2}{\pi} \sum_{n=1, n \neq k}^{N} F_{n} \arcsin \left(\frac{a_{k}}{r_{k, n}}\right) \text { for } k=1,2, \ldots, N,
$$

where $r_{k, n}\left(\geqslant a_{k}+a_{n}\right)$ is the distance between the centers of the $k$ th and the $n$th droplets shown in Fig. 1(a) and given by

$$
r_{k, n}=\sqrt{\left(x_{n}-x_{k}\right)^{2}+\left(y_{n}-y_{k}\right)^{2}} .
$$

Wray et al. [48] also showed that, to the same high degree of accuracy, the local evaporative flux $J_{k}$ is given by

$$
J_{k}(r, \phi)=\mathcal{J}_{k}(r)\left[1-\sum_{n=1, n \neq k}^{N} \frac{F_{n} \sqrt{r_{k, n}^{2}-a_{k}^{2}}}{2 \pi\left(r^{2}+r_{k, n}^{2}-2 r r_{k, n} \cos \left(\phi-\psi_{k, n}\right)\right)}\right],
$$


where

$$
\mathcal{J}_{k}(r)=\frac{2}{\pi \sqrt{a_{k}^{2}-r^{2}}}
$$

is the local evaporative flux from the $k$ th droplet in isolation, and $\psi_{k, n}$ is the angle between the $x$ axis and the line joining the centers of the $k$ th and the $n$th droplets, also shown in Fig. 1(a) and given by

$$
\tan \psi_{k, n}=\frac{y_{n}-y_{k}}{x_{n}-x_{k}}
$$

\section{B. The hydrodynamic problem}

The velocity and pressure within the $k$ th droplet, denoted by $\mathbf{u}_{k}=\mathbf{u}_{k}(r, \phi, z, t)=u_{k} \mathbf{e}_{r}+v_{k} \mathbf{e}_{\phi}+$ $w_{k} \mathbf{e}_{z}$ and $p_{k}=p_{k}(r, \phi, z, t)$, where $t$ denotes time, satisfy the usual mass-conservation and Stokes equations subject to the usual boundary conditions, and the free surface, contact angle and volume of the $k$ th droplet are denoted by $z=h_{k}=h_{k}(r, t), \theta_{k}=\theta_{k}(t)(\ll 1)$ and $V_{k}=V_{k}(t)$, respectively.

We scale and nondimensionalize variables appropriately for the droplet according to

$$
\begin{gathered}
z=\theta_{\text {ref }} a_{\text {ref }} \hat{z}, \quad r=a_{\text {ref }} \hat{r}, \quad t=t_{\text {ref }} \hat{t}, \quad h_{k}=\theta_{\text {ref }} a_{\text {ref }} \hat{h}_{k}, \quad V_{k}=\theta_{\text {ref }} a_{\text {ref }}^{3} \hat{V}_{k}, \\
u_{k}=u_{\text {ref }} \hat{u}_{k}, \quad v_{k}=u_{\text {ref }} \hat{v}_{k}, \quad w_{k}=\theta_{\text {ref }} u_{\text {ref }} \hat{w}_{k}, \quad p_{k}-p_{\mathrm{a}}=\frac{\gamma \theta_{\text {ref }}}{a_{\text {ref }}} \hat{p}_{k},
\end{gathered}
$$

in which $\theta_{\text {ref }}(\ll 1)$ is a characteristic contact angle of the droplets, $\gamma$ is the constant coefficient of surface tension of the fluid, $p_{\mathrm{a}}$ is the constant atmospheric pressure, and $t_{\text {ref }}$ and $u_{\text {ref }}$ are a characteristic time for the evaporation and a characteristic velocity, defined by

$$
t_{\mathrm{ref}}=\frac{\rho \theta_{\mathrm{ref}} a_{\mathrm{ref}}^{2}}{D^{(\mathrm{v})}\left(c_{\mathrm{sat}}^{(\mathrm{v})}-c_{\infty}^{(\mathrm{v})}\right)}, \quad u_{\mathrm{ref}}=\frac{a_{\mathrm{ref}}}{t_{\mathrm{ref}}}=\frac{D^{(\mathrm{v})}\left(c_{\mathrm{sat}}^{(\mathrm{v})}-c_{\infty}^{(\mathrm{v})}\right)}{\rho \theta_{\mathrm{ref}} a_{\mathrm{ref}}},
$$

respectively, where $\rho$ is the constant density of the fluid.

At leading order in $\theta_{\text {ref }} \ll 1$ the governing equations for the $k$ th droplet are, with the hats dropped for clarity,

$$
\frac{1}{r} \frac{\partial\left(r u_{k}\right)}{\partial r}+\frac{1}{r} \frac{\partial v_{k}}{\partial \phi}+\frac{\partial w_{k}}{\partial z}=0, \quad \mathrm{Ca} \frac{\partial^{2} u_{k}}{\partial z^{2}}=\frac{\partial p_{k}}{\partial r}, \quad \mathrm{Ca} \frac{\partial^{2} v_{k}}{\partial z^{2}}=\frac{1}{r} \frac{\partial p_{k}}{\partial \phi}, \quad \frac{\partial p_{k}}{\partial z}=0,
$$

where $\mathrm{Ca}$ is an appropriate capillary number, defined by

$$
\mathrm{Ca}=\frac{\mu u_{\mathrm{ref}}}{\gamma \theta_{\text {ref }}^{3}}=\frac{\mu D^{(\mathrm{v})}\left(c_{\mathrm{sat}}^{(\mathrm{v})}-c_{\infty}^{(\mathrm{v})}\right)}{\gamma \rho \theta_{\mathrm{ref}}^{4} a_{\mathrm{ref}}},
$$

where $\mu$ is the constant viscosity of the fluid. Equation (10) is to be solved subject to zero velocity at the substrate,

$$
u_{k}=0, \quad v_{k}=0, \quad w_{k}=0 \quad \text { at } \quad z=0,
$$

balances of normal and tangential stress at the free surface of the droplet,

$$
p_{k}=-\frac{1}{r} \frac{\partial}{\partial r}\left(r \frac{\partial h_{k}}{\partial r}\right), \quad \frac{\partial u_{k}}{\partial z}=0, \quad \frac{\partial v_{k}}{\partial z}=0 \quad \text { at } \quad z=h_{k},
$$

and the kinematic condition,

$$
\frac{\partial h_{k}}{\partial t}+\frac{1}{r} \frac{\partial}{\partial r}\left(r Q_{k}^{(r)}\right)+\frac{1}{r} \frac{\partial}{\partial \phi}\left(Q_{k}^{(\phi)}\right)=-J_{k} \quad \text { at } \quad z=h_{k}
$$


where $Q_{k}^{(r)}=Q_{k}^{(r)}(r, \phi, t)$ and $Q_{k}^{(\phi)}=Q_{k}^{(\phi)}(r, \phi, t)$, defined by

$$
Q_{k}^{(r)}=\int_{0}^{h_{k}} u_{k} d z, \quad Q_{k}^{(\phi)}=\int_{0}^{h_{k}} v_{k} d z
$$

are the local radial and azimuthal volume fluxes of fluid within the droplet.

We consider the situation in which capillary effects are strong, corresponding to small values of the capillary number $\mathrm{Ca}$, and so we seek asymptotic solutions of the form

$$
u_{k}=u_{k 0}+\mathrm{Ca} u_{k 1}+O\left(\mathrm{Ca}^{2}\right), \quad v_{k}=v_{k 0}+\mathrm{Ca} v_{k 1}+O\left(\mathrm{Ca}^{2}\right), \quad p_{k}=p_{k 0}+\mathrm{Ca} p_{k 1}+O\left(\mathrm{Ca}^{2}\right)
$$

in the limit $\mathrm{Ca} \rightarrow 0$. As we shall see, for the analysis of particle transport presented in Sec. II C, we require only the leading order velocity components $u_{k 0}$ and $v_{k 0}$, which in turn require $p_{k 0}$ and $p_{k 1}$.

At leading order in $\mathrm{Ca} \ll 1$, Eqs. (10) and (13) show that the leading-order pressure is independent of $r, \phi$ and $z$, and is given by $p_{k 0}=p_{k 0}(t)=2 \theta_{k} / a_{k}$, and the leading-order free surface $z=h_{k}(r, t)$ takes the familiar paraboloidal form

$$
h_{k}=\frac{\theta_{k}\left(a_{k}^{2}-r^{2}\right)}{2 a_{k}} \text {. }
$$

The leading-order volume of the droplet is therefore given by

$$
V_{k}=\int_{\phi=0}^{\phi=2 \pi} \int_{r=0}^{r=a_{k}} r h_{k}(r, t) d r d \phi=\frac{\pi \theta_{k} a_{k}^{3}}{4} .
$$

At first order in $\mathrm{Ca} \ll 1$, Eqs. (10), (13), and (12) lead to

$$
p_{k 1}=p_{k 1}(r, \phi, t), \quad u_{k 0}=\frac{p_{k 1 r}}{2}\left(z^{2}-2 h_{k} z\right), \quad v_{k 0}=\frac{p_{k 1 \phi}}{2 r}\left(z^{2}-2 h_{k} z\right),
$$

and so the leading-order local fluid fluxes given by Eq. (15) are

$$
Q_{k}^{(r)}=-\frac{h_{k}^{3}}{3} p_{k 1_{r}}, \quad Q_{k}^{(\phi)}=-\frac{h_{k}^{3}}{3} \frac{p_{k 1_{\phi}}}{r} .
$$

Dropping the subscript "1" on $p_{k 1}$ henceforth for clarity, the kinematic condition given by Eq. (14) therefore gives

$$
\frac{\partial h_{k}}{\partial t}+\frac{1}{r} \frac{\partial}{\partial r}\left(-\frac{r h_{k}^{3}}{3} p_{k_{r}}\right)+\frac{1}{r} \frac{\partial}{\partial \phi}\left(-\frac{h_{k}^{3}}{3} \frac{p_{k_{\phi}}}{r}\right)=-J_{k} .
$$

We may use this condition to obtain the differential equation satisfied by $p_{k}$ by noting that $h_{k}=$ $h_{k}(r, t)$ is independent of the azimuthal coordinate $\phi$, and that, by global mass conservation, the volume $V_{k}$ satisfies

$$
\frac{d V_{k}}{d t}=-\int_{\phi=0}^{\phi=2 \pi} \int_{r=0}^{r=a_{k}} r J_{k} d r d \phi
$$

so that, with Eqs. (17) and (18), $\partial h_{k} / \partial t$ may be written as

$$
\frac{\partial h_{k}}{\partial t}=\frac{d \theta_{k}}{d t} \frac{a_{k}^{2}-r^{2}}{2 a_{k}}=\frac{4}{\pi a_{k}^{3}} \frac{d V_{k}}{d t} \frac{a_{k}^{2}-r^{2}}{2 a_{k}}=-\frac{2\left(a_{k}^{2}-r^{2}\right)}{\pi a_{k}^{4}} \int_{\phi=0}^{\phi=2 \pi} \int_{r=0}^{r=a_{k}} r J_{k} d r d \phi .
$$

Finally, therefore, the kinematic condition given by Eq. (21) may be expressed in the form

$$
\frac{1}{r} \frac{\partial}{\partial r}\left(\frac{r h_{k}^{3}}{3} p_{k r}\right)+\frac{h_{k}^{3}}{3 r^{2}} p_{k \phi \phi}=J_{k}-\frac{2\left(a_{k}^{2}-r^{2}\right)}{\pi a_{k}^{4}} \int_{\phi=0}^{\phi=2 \pi} \int_{r=0}^{r=a_{k}} r J_{k} d r d \phi,
$$

which is a partial differential equation for $p_{k}$, with all of the other quantities in Eq. (24) being known. Once $p_{k}$ is determined from Eq. (24), the local fluid fluxes $Q_{k}^{(r)}$ and $Q_{k}^{(\phi)}$ are given by Eq. (20). 
The depth-averaged radial and azimuthal velocities, denoted by $\bar{u}_{k}=\bar{u}_{k}(r, \phi, t)$ and $\bar{v}_{k}=$ $\bar{v}_{k}(r, \phi, t)$, are defined by

$$
\bar{u}_{k}=\frac{1}{h_{k}} \int_{0}^{h_{k}} u_{k} d z=\frac{Q_{k}^{(r)}}{h_{k}}, \quad \bar{v}_{k}=\frac{1}{h_{k}} \int_{0}^{h_{k}} v_{k} d z=\frac{Q_{k}^{(\phi)}}{h_{k}},
$$

respectively. For future reference, note that the streamlines of the depth-averaged flow are determined by solving

$$
\frac{d r}{d \phi}=\frac{r \bar{u}_{k}}{\bar{v}_{k}}=\frac{r Q_{k}^{(r)}}{Q_{k}^{(\phi)}}=\frac{r^{2} p_{k r}}{p_{k \phi}} .
$$

Since $\bar{u}_{k}$ and $\bar{v}_{k}$ have the same functional dependence on $h$, the $\theta_{k}$ has canceled out of Eq. (26), and so the streamlines depend on time only via changes in the flux $J_{k}$. This means that in certain situations the computation of the streamlines is simplified somewhat by the fact (mentioned earlier) that $J_{k}$ and hence $F_{k}$ are independent of $t$ except for discontinuous jumps when any droplet completely evaporates. Specifically, if the droplets are arranged in such a way that all of them completely evaporate at exactly the same time, then their streamlines remain unchanged throughout the evaporation. In this case the determination of the density of the deposit reduces to performing a single integral, as will be described in Sec. IV B below.

\section{The particle-transport problem}

The motion of the particles suspended within each droplet is due to a combination of advection by the flow and diffusion, and so the concentration of particles in the $k$ th droplet, denoted by $c_{k}=$ $c_{k}(r, \phi, z, t)$, satisfies the (scaled) advection-diffusion equation

$$
\theta_{\text {ref }}^{2} \operatorname{Pe}\left[\frac{\partial c_{k}}{\partial t}+u_{k} \frac{\partial c_{k}}{\partial r}+\frac{v_{k}}{r} \frac{\partial c_{k}}{\partial \phi}+w_{k} \frac{\partial c_{k}}{\partial z}\right]=\theta_{\text {ref }}^{2}\left[\frac{1}{r} \frac{\partial}{\partial r}\left(r \frac{\partial c_{k}}{\partial r}\right)+\frac{1}{r^{2}} \frac{\partial^{2} c_{k}}{\partial \phi^{2}}\right]+\frac{\partial^{2} c_{k}}{\partial z^{2}},
$$

in which $c_{k}$ has been nondimensionalized according to $c_{k}=c_{\text {ref }} c_{k}^{*}$, where $c_{\text {ref }}$ is a characteristic concentration of particles, $\mathrm{Pe}=u_{\mathrm{ref}} a_{\mathrm{ref}} / D^{(\mathrm{p})}$ is an appropriate Péclet number, and $D^{(\mathrm{p})}$ is the constant diffusion coefficient for the particles in the fluid, and where the star subscript has again been dropped for clarity. Equation (27) is subject to conditions of no flux of particles through either the free surface of the droplet or the substrate,

$$
\mathbf{n}_{k} \cdot \nabla c_{k}=\operatorname{Pe} c_{k} J_{k} \quad \text { at } \quad z=h_{k}, \quad \frac{\partial c_{k}}{\partial z}=0 \quad \text { at } \quad z=0,
$$

where $\mathbf{n}_{k}$ denotes the outward unit normal to the free surface of the $k$ th droplet.

As is well known (see, for example, Ref. [22]), when the Péclet number Pe is such that $\theta_{\text {ref }}^{2} \ll$ $\theta_{\text {ref }}^{2} \mathrm{Pe} \ll 1$, the leading-order concentration of particles, $c_{k}=c_{k}(r, \phi, t)$, is independent of $z$ and satisfies

$$
\frac{\partial c_{k}}{\partial t}+\bar{u}_{k} \frac{\partial c_{k}}{\partial r}+\frac{\bar{v}_{k}}{r} \frac{\partial c_{k}}{\partial \phi}=\frac{c_{k} J_{k}}{h_{k}}
$$

where the depth-averaged radial and azimuthal velocities $\bar{u}_{k}$ and $\bar{v}_{k}$ are given by Eq. (25). Equation (29) may be solved by the method of characteristics:

$$
\frac{d c_{k}}{d t}=\frac{c_{k} J_{k}}{h_{k}} \quad \text { on the characteristics } \quad \frac{d r}{d t}=\bar{u}_{k} \quad \text { and } \quad \frac{d \phi}{d t}=\frac{\bar{v}_{k}}{r},
$$

subject to a prescribed initial condition $c_{k}=c_{k}(r, \phi, 0)$ at $t=0$. For simplicity, in all of the results presented below we assume that the initial concentration of particles takes the same uniform value in all of the droplets, which we may, without loss of generality, take to be unity. 


\section{SOLUTION FOR THE PRESSURE $p_{k}$}

In general, the expression for the evaporative flux $J_{k}$ given by Eq. (5) is rather complicated, and precludes solving Eq. (24) for the pressure $p_{k}$ in closed form; however, we may determine $p_{k}$ to arbitrary accuracy as follows.

For $r \leqslant a_{k}\left(<r_{k, n}\right)$ we expand $J_{k}$ given by Eq. (5) as the convergent series

$$
J_{k}(r, \phi)=\mathcal{J}_{k}(r)\left[1-\sum_{n=1, n \neq k}^{N} \frac{F_{n}}{2 \pi r_{k, n}}\left(1+\frac{2 r \cos \left(\phi-\psi_{k, n}\right)}{r_{k, n}}+\cdots\right)\right],
$$

which we may rearrange in the form of a truncated Fourier series

$$
J_{k}(r, \phi)=\mathcal{J}_{k}(r)\left[j_{0}(r)+\sum_{n=1, n \neq k}^{N} \sum_{m=1}^{M} j_{n, m}(r) \cos \left[m\left(\phi-\psi_{k, n}\right)\right]\right]
$$

for a chosen number of modes $M(M=1,2,3, \ldots)$, with known functions $j_{0}=j_{0}(r)$ and $j_{n, m}=$ $j_{n, m}(r)$. To determine $p_{k}$ we decompose it into a corresponding form, namely,

$$
p_{k}=p_{k}^{(0)}(r)+\sum_{n=1, n \neq k}^{N} \sum_{m=1}^{M} p_{k}^{(n, m)}(r) \cos \left[m\left(\phi-\psi_{k, n}\right)\right],
$$

substitution of which into Eq. (24) leads to a sequence of differential equations for $p_{k}^{(0)}$ and $p_{k}^{(n, m)}$,

$$
\frac{1}{r} \frac{d}{d r}\left(\frac{r h_{k}^{3}}{3} \frac{d p_{k}^{(0)}}{d r}\right)=\mathcal{J}_{k}(r) j_{0}(r)-\frac{4 F_{k}}{\pi \theta_{k} a_{k}^{3}} \frac{a_{k}^{2}-r^{2}}{2 a_{k}}
$$

and

$$
\frac{1}{r} \frac{d}{d r}\left(\frac{r h_{k}^{3}}{3} \frac{d p_{k}^{(n, m)}}{d r}\right)-\frac{m^{2} h_{k}^{3}}{3 r^{2}} p_{k}^{(n, m)}=\mathcal{J}_{k}(r) j_{n, m}(r) .
$$

Equation (34) may be solved directly (up to an irrelevant additive constant) subject to regularity at the origin. Equation (35) may be solved by the method of variation of parameters. Specifically, the homogeneous version of Eq. (35), namely,

$$
\frac{d^{2} p_{k}^{(n, m)}}{d r^{2}}+\left(\frac{1}{r}-\frac{6 r}{a_{k}^{2}-r^{2}}\right) \frac{d p_{k}^{(n, m)}}{d r}-\frac{m^{2}}{r^{2}} p_{k}^{(n, m)}=0,
$$

has solutions $p_{k}^{(n, m)}=P_{k 1}^{(m)}(r)$ and $p_{k}^{(n, m)}=P_{k 2}^{(m)}(r)$ given by

$$
P_{k 1}^{(m)}=\left(\frac{r}{a_{k}}\right)^{m}{ }_{2} F_{1}\left(\frac{1}{2}\left(3+M_{+}\right), \frac{1}{2}\left(3+M_{-}\right) ; m+1 ; \frac{r^{2}}{a_{k}^{2}}\right),
$$

which satisfies

$$
P_{k 1}^{(m)} \sim \begin{cases}\left(\frac{r}{a_{k}}\right)^{m} & \text { for } \frac{r}{a_{k}} \ll 1, \\ \frac{\Gamma(1+m)}{4 \Gamma\left(\frac{1}{2}\left(3+M_{-}\right)\right) \Gamma\left(\frac{1}{2}\left(3+M_{+}\right)\right)}\left(\frac{a_{k}}{a_{k}-r}\right)^{2} & \text { for } 1-\frac{r}{a_{k}} \ll 1,\end{cases}
$$

and

$$
P_{k 2}^{(m)}=\left(\frac{a_{k}}{r}\right)^{m}{ }_{2} F_{1}\left(\frac{1}{2}\left(3-M_{+}\right), \frac{1}{2}\left(3-M_{-}\right) ; 3 ; 1-\frac{r^{2}}{a_{k}^{2}}\right),
$$


which satisfies

$$
P_{k 2}^{(m)} \sim\left\{\begin{array}{lc}
\frac{2 \Gamma(m)}{\Gamma\left(\frac{1}{2}\left(3+M_{-}\right)\right) \Gamma\left(\frac{1}{2}\left(3+M_{+}\right)\right)}\left(\frac{a_{k}}{r}\right)^{m} & \text { for } \frac{r}{a_{k}} \ll 1, \\
1 & \text { for } 1-\frac{r}{a_{k}} \ll 1,
\end{array}\right.
$$

where $M_{ \pm}=m \pm \sqrt{m^{2}+9}$, and so the solution of the inhomogeneous equation (35) is

$$
p_{k}^{(n, m)}(r)=3 P_{k 2}^{(m)}(r) \int_{0}^{r} \frac{\mathcal{J}_{k}(\tilde{r}) j_{n, m}(\tilde{r}) P_{k 1}^{(m)}(\tilde{r})}{W^{(m)}(\tilde{r}) h_{k}(\tilde{r})^{3}} d \tilde{r}+3 P_{k 1}^{(m)}(r) \int_{r}^{a_{k}} \frac{\mathcal{J}_{k}(\tilde{r}) j_{n, m}(\tilde{r}) P_{k 2}^{(m)}(\tilde{r})}{W^{(m)}(\tilde{r}) h_{k}(\tilde{r})^{3}} d \tilde{r}
$$

where

$$
W^{(m)}=P_{k 1}^{(m)} \frac{d P_{k 2}^{(m)}}{d r}-P_{k 2}^{(m)} \frac{d P_{k 1}^{(m)}}{d r}
$$

is the Wronskian, which can be evaluated to give

$$
W^{(m)}=-\frac{4 \Gamma(m+1) a_{k}^{6}}{\Gamma\left(\frac{1}{2}\left(3+M_{+}\right)\right) \Gamma\left(\frac{1}{2}\left(3+M_{-}\right)\right) r\left(a_{k}^{2}-r^{2}\right)^{3}} .
$$

Note that we have chosen the forms of the homogeneous solutions and imposed the boundary conditions by selecting the lower limits of the integrals in Eq. (41) so as to ensure regularity at the origin (first integral) and at the contact line (second integral). In general, the integrals in Eq. (41) must be evaluated numerically to obtain $p_{k}^{(n, m)}(r)$. Note, however, that, as described in the Appendix, even greater analytical progress is possible for the corresponding problem in the limit of large Bond number.

\section{A PAIR OF IDENTICAL DROPLETS}

In this Section we apply the general methodology developed in Secs. II and III to determine the densities of the deposits from a pair of identical droplets, a situation for which the predictions of the present asymptotic theory for the local evaporative flux $J_{k}$ and the integral evaporative flux $F_{k}$ were validated by Wray et al. [48]. In Sec. IV A we determine the local evaporative fluxes, the fluid fluxes, and the resulting streamlines of the depth-averaged flows, in Sec. IV B we determine the density of the deposit, while in Sec. IV C we compare the theoretical predictions for the density of the deposit with the experimental results of Pradhan and Panigrahi [39].

\section{A. Evaporative fluxes, fluid fluxes, and streamlines}

Consider the evaporation of a pair of identical droplets, which we may, without loss of generality, take to be of unit radius $a_{1}=a_{2}=1$, with their centers located at $( \pm b / 2,0)$, i.e., with their centers a distance $r_{1,2}=r_{2,1}=b(>2)$ apart. As Wray et al. [48] showed, the two droplets have the same integral evaporative flux given by $F_{1}=F_{2}=F$, where

$$
F=\frac{4}{1+(2 / \pi) \arcsin (1 / b)}
$$

By symmetry, it is sufficient to consider only the left-hand droplet with its center located at $(-b / 2,0)$, corresponding to $k=1$. The local evaporative flux from the surface of the droplet is given by

$$
J_{1}=\mathcal{J}(r)\left[1-\frac{F \sqrt{b^{2}-1}}{2 \pi\left(r^{2}+b^{2}-2 r b \cos \phi\right)}\right]
$$




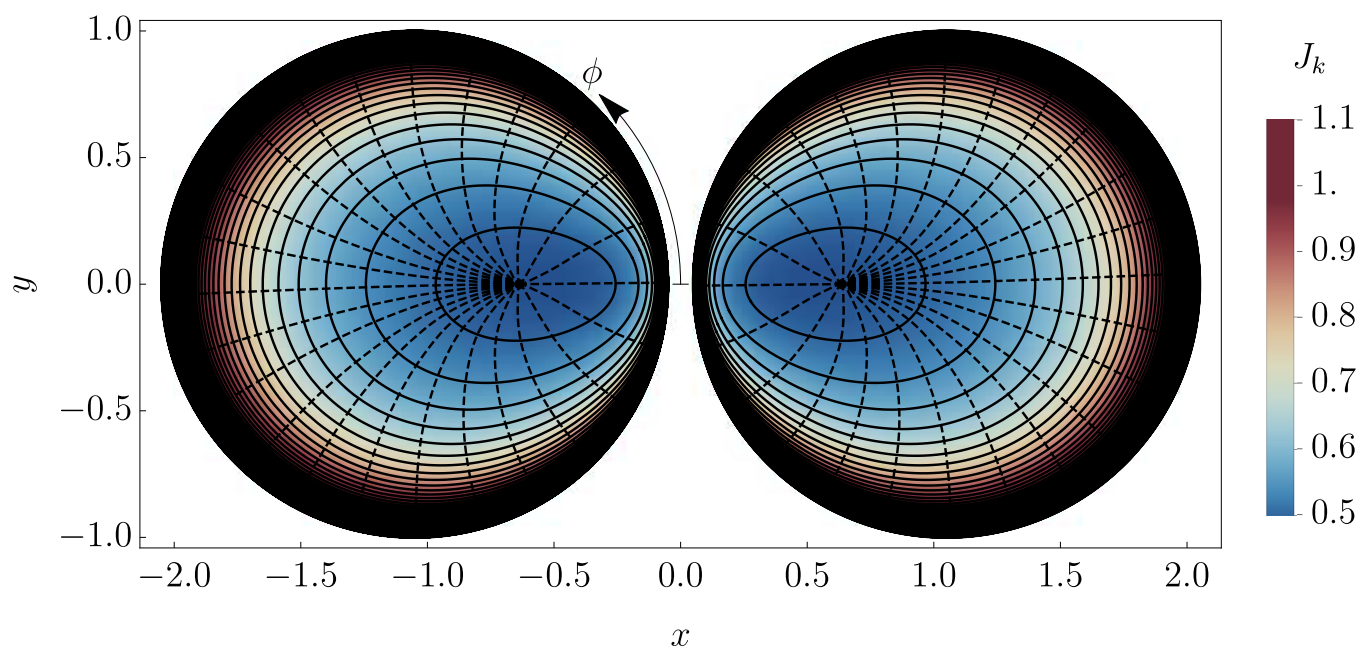

FIG. 2. Contours of the local evaporative fluxes $J_{1}$ (left-hand droplet) and $J_{2}$ (right-hand droplet) (solid curves), and the resulting streamlines of the depth-averaged flows (dashed curves) for a pair of identical droplets of unit radius with their centers a distance $b=2.1$ apart.

which may be expanded as

$$
J_{1}=\mathcal{J}(r) \sum_{m=0}^{M} j_{m}(r) \cos m \phi
$$

where

$$
j_{0}=1+\frac{F\left(1-2 b^{2}-2 r^{2}\right)}{4 \pi b^{3}}, \quad j_{1}=\frac{F\left(1-2 b^{2}-2 r^{2}\right) r}{2 \pi b^{4}}, \quad j_{2}=-\frac{F r^{2}}{\pi b^{3}}, \quad j_{3}=-\frac{F r^{3}}{\pi b^{4}}, \quad \ldots
$$

and

$$
\mathcal{J}(r)=\frac{2}{\pi \sqrt{1-r^{2}}}
$$

is the flux from the same droplet in isolation. The expansion given by Eq. (46) with Eq. (47) can then be used to determine the pressure $p_{1}$ in Eq. (33), and thus the local fluid fluxes $Q_{1}^{(r)}$ and $Q_{1}^{(\phi)}$ in Eq. (20). Figure 2 shows contours of the local evaporative fluxes $J_{1}$ (left-hand droplet) and $J_{2}$ (right-hand droplet), and the resulting streamlines of the depth-averaged flows for $b=2.1$, with the expansion given by Eq. (46) truncated after $M=10$ Fourier modes. Figure 2 illustrates how the shielding effect reduces the local evaporative flux the most where the droplets are closest together (i.e., at $\phi=0$ ) and the least where they are furthest apart (i.e., at $\phi=\pi$ ) [48]. For an isolated droplet the contours of $\mathcal{J}$ given by Eq. (48) are concentric circles and the streamlines are radial lines; Fig. 2 also illustrates how the shielding effect skews both of them toward the other droplet.

\section{B. Density of the deposit}

We now determine the density of the deposit resulting from the evaporation of the droplets. By symmetry, the two droplets completely evaporate at the same time, and so the streamlines of their depth-averaged flows remain unchanged throughout the evaporation and, as in Sec. IV A, it is sufficient to consider only the left-hand droplet with its center located at $(-b / 2,0)$.

We work relative to (nonorthogonal) curvilinear coordinates $(\chi, \xi)$ defined by the streamlines with their origin at the "source" from which all of the streamlines of the depth-averaged flow 
emanate, denoted by $\left(x_{\mathrm{S}}, y_{\mathrm{S}}\right)$, shown in Fig. 2. Note that, contrary to what Fig. 2 may suggest, the source from which the streamlines emanate, $\left(x_{\mathrm{S}}, y_{\mathrm{S}}\right)$, does not, in general, coincide exactly with the location of the minimum of the local evaporative flux. The difference between the two is readily evident in Fig. 6 which appears subsequently in Sec. V. The coordinate $\xi \in[0,2 \pi$ ) parameterizes the streamlines such that the local behavior of the streamlines near the source is given by $(x, y)=\left(x_{\mathrm{S}}, y_{\mathrm{S}}\right)+\delta(\cos \xi, \sin \xi)+O\left(\delta^{2}\right)$ as $\delta \rightarrow 0^{+}$, while the coordinate $\chi(\geqslant 0)$ is the arc length along each streamline, measuring from $\chi=0$ at the source to $\chi=\chi_{\max }(\xi)$ at the contact line. Note that $\chi_{\max }(\xi)$ is therefore the length of the streamline parameterized by $\xi$ from the source to the contact line.

Since the fluid flow always advects the particles along the streamlines toward the contact line, the mass of the deposit that eventually accumulates at the contact line between the point with coordinate $\xi=0$ and a general point with coordinate $\xi$, denoted by $M=M(\xi)$, is exactly equal to the mass of particles originally in the curved sector between the streamlines parameterized by $\xi=0$ and by $\xi$, i.e.,

$$
M(\xi)=\int_{\hat{\xi}=0}^{\hat{\xi}=\xi} \int_{\chi=0}^{\chi=\chi_{\max }(\hat{\xi})} h[x(\chi, \hat{\xi}), y(\chi, \hat{\xi})] \frac{\partial(x, y)}{\partial(\chi, \hat{\xi})} d \chi d \hat{\xi} .
$$

Note that, by definition, $M(0)=0$ and $M(2 \pi)=\pi / 4$. Once $M$ has been determined, the density of the deposit at a point on the contact line with polar angle $\phi$, denoted by $D=D(\phi)$, is then given by

$$
D=\left.\frac{1}{r} \frac{d M}{d \phi}\right|_{r=1}=\left.\frac{1}{r} \frac{d M}{d \xi} \frac{\partial \xi}{\partial \phi}\right|_{r=1} .
$$

Figure 3 shows the evaporative flux at the contact line normalized by the corresponding flux for the same droplet in isolation, $\lim _{r \rightarrow 1^{-}} J_{1}(r, \phi) / \mathcal{J}(r)$, and the density of the deposit $D$ as functions of the azimuthal coordinate $\phi$ for several values of $b$. In particular, Fig. 3(b) shows that the shielding effect described in Sec. IV A and shown in Fig. 2 leads to a spatially nonuniform deposit with the smallest density where the shielding effect is strongest (i.e., at $\phi=0$ ) and the largest density where it is weakest (i.e., at $\phi=\pi$ ). Note that, by conservation of mass, the total mass of the deposit is the same for all of the values of $b$ used in Fig. 3(b).

\section{Comparison with experimental results}

In general, comparing theoretical predictions for the density of a deposit with experimentally obtained images of deposition patterns is a challenging task. In particular, because the depth of the deposit cannot usually be readily determined from images taken from above the droplet, obtaining a quantitative measure of the amount of deposit from experimental images may often not be possible due to saturation and nonlinearity of the data. For example, in Fig. 2 of Pradhan and Panigrahi [39], reproduced here as Fig. 4, the image of the final deposition pattern from a pair of similar droplets of water containing $1 \mu \mathrm{m}$ particles shown in Fig. 4(d) has zero transmittance through the contact-line deposit, making it impossible to quantify its spatial distribution. However, the image of the final deposition pattern from a pair of similar droplets of ink shown in Fig. 4(b) has nonzero transmittance throughout the vast majority of the contact-line deposit, and so offers us an opportunity to quantify the relative amount of deposit as a function of azimuthal position.

To compare the results of Pradhan and Panigrahi [39] with the present theoretical predictions, it is first necessary to convert the information contained within the image into a form proportional to the density distribution $D$. The raw data extracted from the image corresponds to the reflectance of the deposit; this must be converted into its absorbance, which can then be related to its concentration via the Beer-Lambert law [50],

$$
A=-\log _{10}\left(\frac{P}{P_{0}}\right)=\epsilon l C
$$



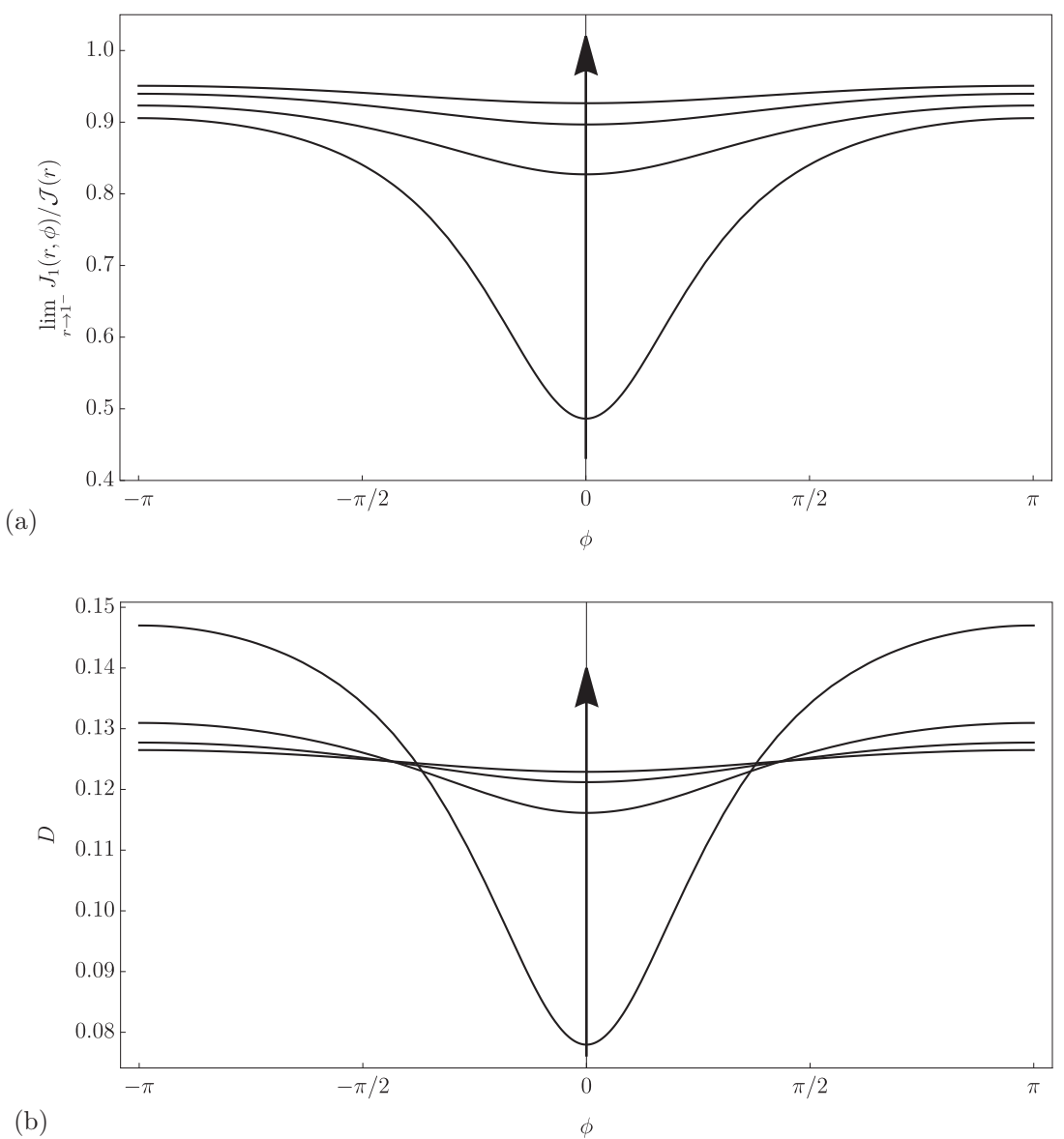

FIG. 3. (a) The normalized evaporative flux at the contact line, $\lim _{r \rightarrow 1^{-}} J_{1}(r, \phi) / \mathcal{J}(r)$, and (b) the density of the deposit $D$ as functions of the azimuthal coordinate $\phi$ for a pair of identical droplets of unit radius with their centers distances $b=2.5,5,7.5,10$ apart. In both parts the arrow indicates the direction of increasing $b$.

where $A$ is the absorbance, $P$ is the radiant power (reflectance) or intensity of the light as measured at each pixel in the image, $P_{0}$ is the initial radiant power of the light before absorbance, $\epsilon$ is the molar absorptivity of the deposit (which may reasonably be assumed to be constant), $l$ is the path length of the light in the deposit, and $C$ is the concentration of the absorbing species. The values for $P$ are known, as this is the light gathered by each pixel in the sensor of the camera to generate the image; however, the value of $P_{0}$ is unknown. (Ideally $P_{0}$ would have been determined through the collection of a reference image in which the light reflected from a calibrated sample, such as a 99\% reflectance standard, was captured.) Therefore, it is necessary to make an estimate of $P_{0}$, and to do this we used the maximum possible brightness value of 255 as the reference value for all of the pixels (consistent with the brightness of the image outside the footprint of the droplets). To gather only density data from the deposit near the contact line of each droplet, and not the density of any residual deposit left within it, the data was taken from an annular region around the edge of the footprint of each droplet. Slightly unfortunately, as Fig. 4 shows, in the published image the deposit from the left-hand droplet is overlaid by the head of an arrow that the authors added to indicate the region of weakest deposition. To reduce artifacts associated with this arrowhead, it was removed from the data by interpolating from the neighboring pixels. The procedure for the extraction of the data for each droplet was implemented in Python [51] and is detailed as follows: 


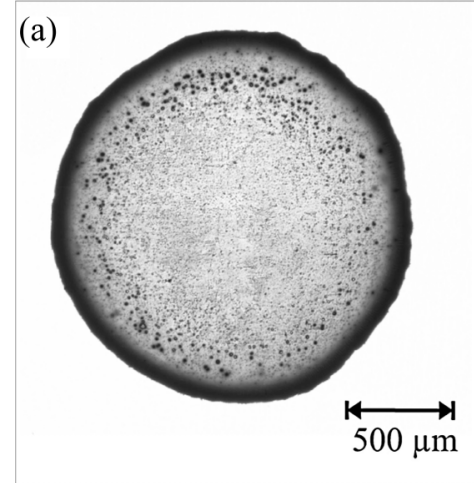

(c)

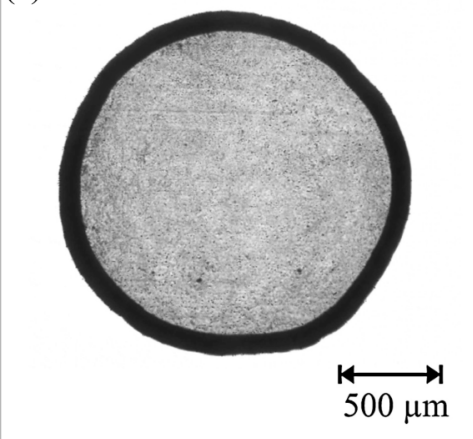

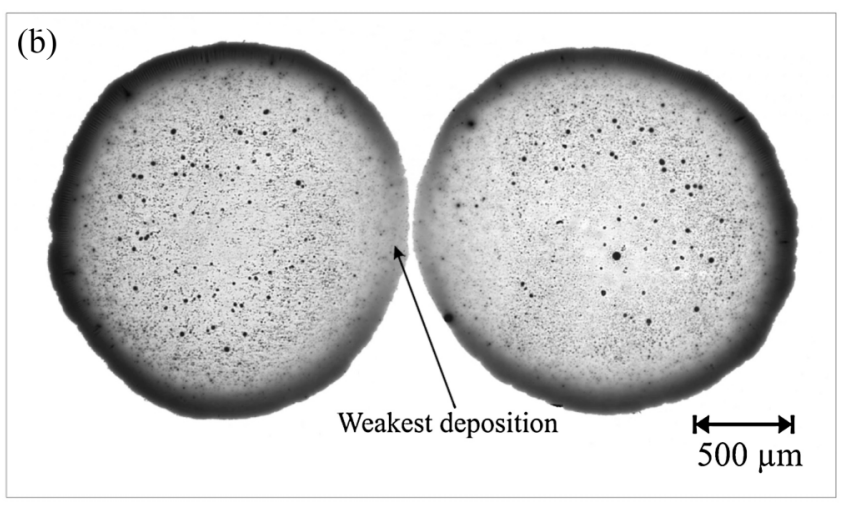

(d)

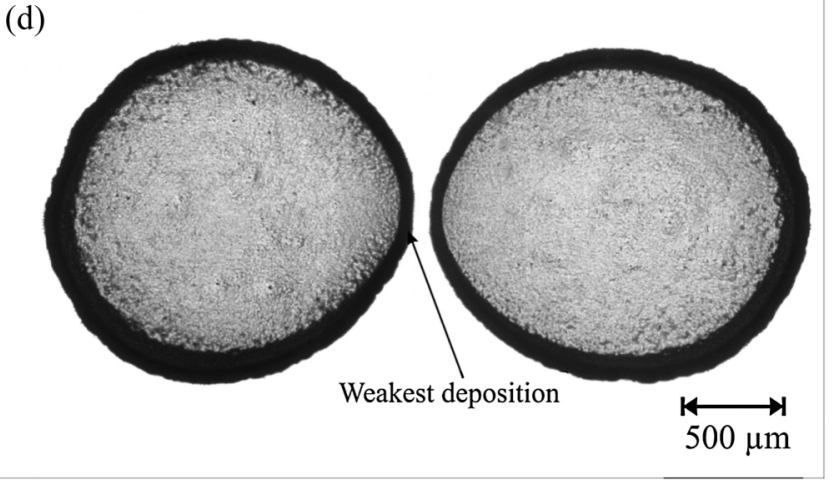

FIG. 4. Figure 2 of Pradhan and Panigrahi [39], showing contact-line deposits from (a) a single droplet and (b) a pair of droplets of ink with a minimum separation of $25 \mu \mathrm{m}$, (c) a single droplet and (d) a pair of droplets of water containing $1 \mu \mathrm{m}$ particles with a minimum separation of $95 \mu \mathrm{m}$. Reprinted from Colloids and Surfaces A: Physicochemical and Engineering Aspects, Volume 482, "Deposition pattern of interacting droplets," pages 562-567, copyright 2015, with permission from Elsevier.

(1) Convert the image to binary and determine its center of mass.

(2) Trace out the outer perimeter of the deposit.

(3) Define a second inner perimeter at $80 \%$ of the radius of the outer perimeter measured relative to the center of mass.

(4) Divide the annular region between the inner and outer perimeters into $N$ sectors subtending equal angles at the center of mass. In practice, $N=300$ sectors were used.

(5) Use the Beer-Lambert law given by Eq. (51) to calculate the absorbance of each pixel, which is proportional to the mass of residue per unit area.

(6) Integrate the mass per unit area numerically over each sector, and divide by the angle subtended, to determine the corresponding proportional mass per unit length of the contact line (i.e., the density to be compared with $D$ ).

The constant of proportionality for comparison with $D$ is determined by imposing the condition that the integral of the density is $\pi / 4$, in line with the nondimensionalization in Sec. IIC. Figure 5 shows a comparison between the present theoretical predictions and the experimental results extracted from Fig. 2(b) of Pradhan and Panigrahi [39] using the procedure described above. Specifically, Fig. 5(a) compares the density of the deposit predicted by Eq. (50) (shown with the black solid curve) with the experimental results for the left-hand droplet (shown with the blue dashed curve) and the right-hand droplet (shown with the red dotted curve). Given the approximations made in both the mathematical model and in the processing of the experimental image, the agreement 

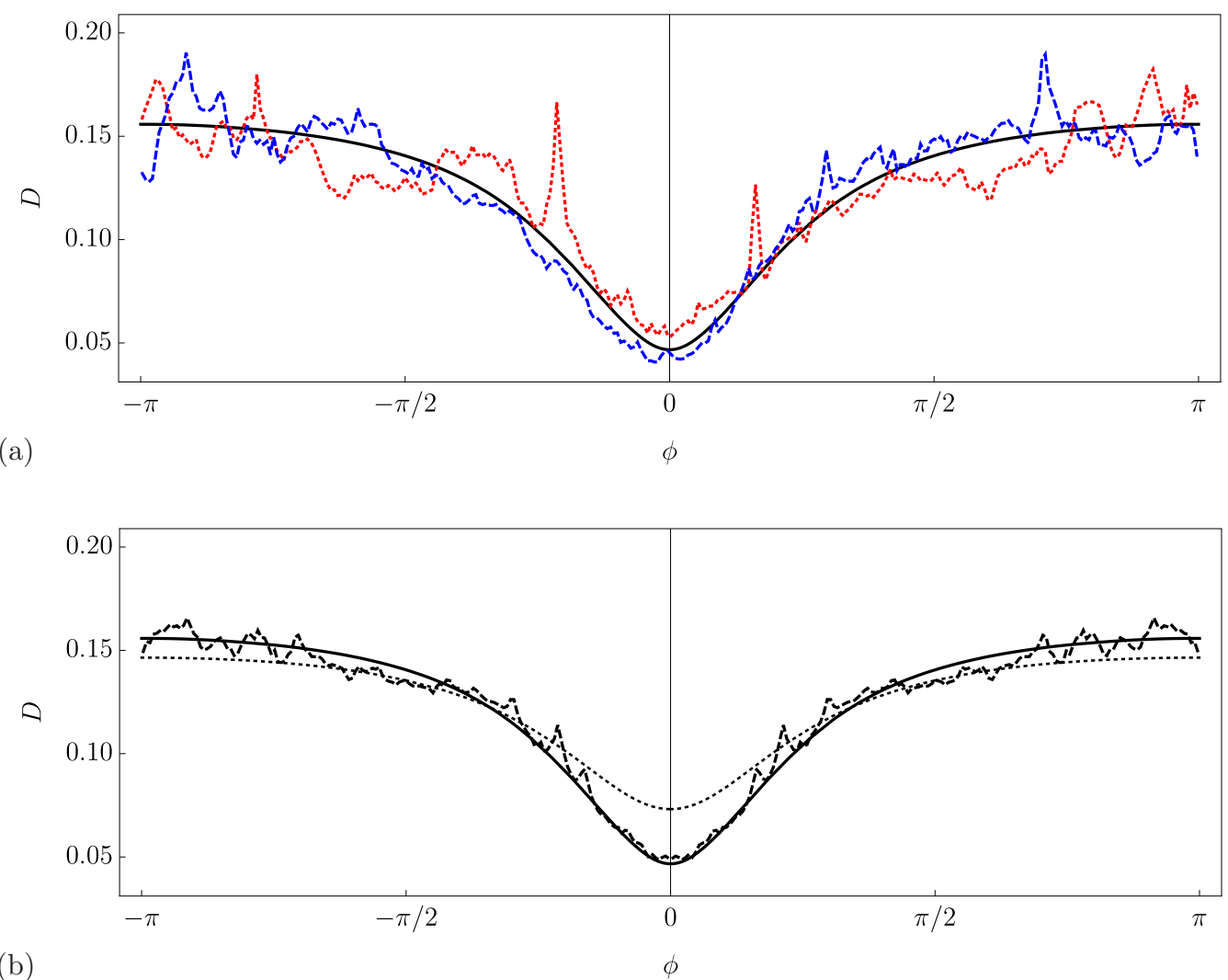

FIG. 5. (a) Comparison between the density of the deposit predicted by Eq. (50) (black solid curve) and the experimental results for the left-hand droplet (blue dashed curve) and the right-hand droplet (red dotted curve) extracted from Fig. 2(b) of Pradhan and Panigrahi [39]. (b) Comparison between the density of the deposit predicted by Eq. (50) (black solid curve) and the averaged experimental results for both droplets shown in part (a) (black dashed curve), and the corresponding phenomenological estimate derived by Wray et al. [48] given by Eq. (53) (black dotted curve).

between theory and experiment shown in Fig. 5(a) is remarkably good, especially when it is noted that no fitting parameters have been used. The only scaling used is to ensure that the total mass (not given by Pradhan and Panigrahi [39]) is the same for theory and experiment.

While the agreement between theory and experiment shown in Fig. 5(a) is already good, with an integral absolute relative error of around $7 \%$ for the left-land droplet and $9 \%$ for the right-hand droplet, the experimental results are inevitably rather noisy. Figure 5(b) compares the density of the deposit predicted by Eq. (50) (shown again with the black solid curve) with the experimental results averaged across both droplets, as well as about $\phi=0$ (shown with the black dashed curve). In particular, Fig. 5(b) shows that, as expected, averaging reduces the noise in the experimental results and, rather pleasingly, leads to even better agreement between theory and experiment than that shown in Fig. 5(a), with an integral absolute relative error of around 3\%. Figure 5(b) also includes the corresponding phenomenological estimate of the radially-integrated fluid flux (now interpreted as the density of the deposit) derived by Wray et al. [48] by using the approach of Sáenz et al. [31], denoted here by $R_{1}(\phi, b)$, given by

$$
R_{1}(\phi, b)=\int_{0}^{1} \mathcal{J}(r)\left[1-\frac{F \sqrt{b^{2}-1}}{2 \pi\left(\rho^{2}+b^{2}-2 b r \cos \phi\right)}\right] r d r
$$


which can be evaluated to yield

$$
R_{1}(\phi, b)=\frac{2}{\pi}\left[1-\frac{F \sqrt{1-k^{2}}}{\pi^{2} \sin \phi} \operatorname{Im}\left\{\frac{\log \left[-\left(k e^{-i \phi}+\sqrt{k^{2} e^{-2 i \phi}-1}\right)\right]}{\sqrt{k^{2} e^{-2 i \phi}-1}}\right\}\right],
$$

where $k=1 / b(<1)$ and $F$ is given by Eq. (44). In particular, Fig. 5(b) shows that the estimate given by Eq. (53) is reasonably accurate, with an integral absolute relative error of around 9\%, and captures the experimental results qualitatively but not quite quantitatively.

\section{A TRIPLET OF IDENTICAL DROPLETS}

In this Section we use the same approach as that described in Sec. IV to determine the densities of the deposits from a triplet of identical droplets of unit radius with their centers located at $(-b / 2,0)$ and $(b / 2)(\cos (\pi / 3), \pm \sin (\pi / 3))$, i.e., with their centers a distance $r_{1,2}=r_{2,3}=r_{3,1}=$ $b \sin (\pi / 3)(>2)$ apart. Note that, in the terminology used by Wray et al. [48], the centers of the droplets lie at the vertices of an equilateral triangle with side $b \sin (\pi / 3)$ and circumradius $b / 2$. Similarly to in Sec. II, by symmetry it is sufficient to consider only the left-most droplet with its center located at $(-b / 2,0)$, corresponding to $k=1$. The results are summarized in Figs. 6 and 7, which show contours of the local evaporative fluxes $J_{k}$ for $k=1,2,3$ and the resulting streamlines of the depth-averaged flows for $b \sin (\pi / 3)=2.1$, and the normalized evaporative flux at the contact line, $\lim _{r \rightarrow 1^{-}} J_{1}(r, \phi) / \mathcal{J}(r)$, and the density of the deposit $D$ as functions of the azimuthal coordinate $\phi$ for several values of $b$, respectively. In particular, Figs. 6 and 7 show that, as expected, the shielding effect again reduces the local evaporative flux and leads to a spatially nonuniform deposit at the contact line. However, in this case the effect is evidently more subtle than that described in Sec. IV for a pair of droplets. Specifically, when the droplets are sufficiently far apart, the shielding effect reduces the local evaporative flux the most in the direction toward the center of the triangle (i.e., at $\phi=0$ ) and the least in the direction away from the center of the triangle (i.e., at $\phi=\pi$ ), but these directions do not now correspond to those in which the droplets are closest together (i.e., at $\phi= \pm \pi / 6$ ). However, when the droplets are sufficiently close together, the local evaporative flux develops two (symmetric) local minima at nonzero values of $\phi$ [as shown by the curve for $b \sin (\pi / 3)=2.1$ in Fig. 7(a)], and the density of the deposit develops local maxima and minima [as shown by the curve for $b \sin (\pi / 3)=2.1$ in Fig. 7(b)]. Note that, as in Fig. 3(b), by conservation of mass, the total mass of the deposit is the same for all of the values of $b$ used in Fig. 7(b).

\section{CONCLUSIONS}

In the present work we obtained theoretical predictions for the spatially nonuniform densities of the contact-line deposits left on the substrate after the competitive diffusion-limited evaporation of multiple thin axisymmetric sessile droplets in proximity to each other. In particular, we gave predictions for the deposits from a pair of identical droplets, which showed that the deposit is reduced the most where the droplets are closest together, and demonstrated excellent quantitative agreement with experimental results of Pradhan and Panigrahi [39]. We also gave corresponding predictions for a triplet of identical droplets arranged in an equilateral triangle, which showed that the effect of shielding on the deposit is more subtle in this case.

We note that, while the present analysis is formally restricted to thin droplets, the fact that much of the deposition from a nonthin droplet occurs toward the end of its lifetime when the contact angle is small and the velocity within the droplet is large (sometime referred to as "the rush hour"; see, for example, Refs. $[16,18]$ ) means that the results of the present analysis are also expected to provide useful predictions for the contact-line deposits from nonthin droplets.

A brief observation about the validity of the diffusion-limited model of evaporation is perhaps appropriate here. The excellent agreement with experimental results of Khilifi et al. [45] found by Wray et al. [48] attests to the accuracy of the theoretical predictions for the integral evaporative flux, 


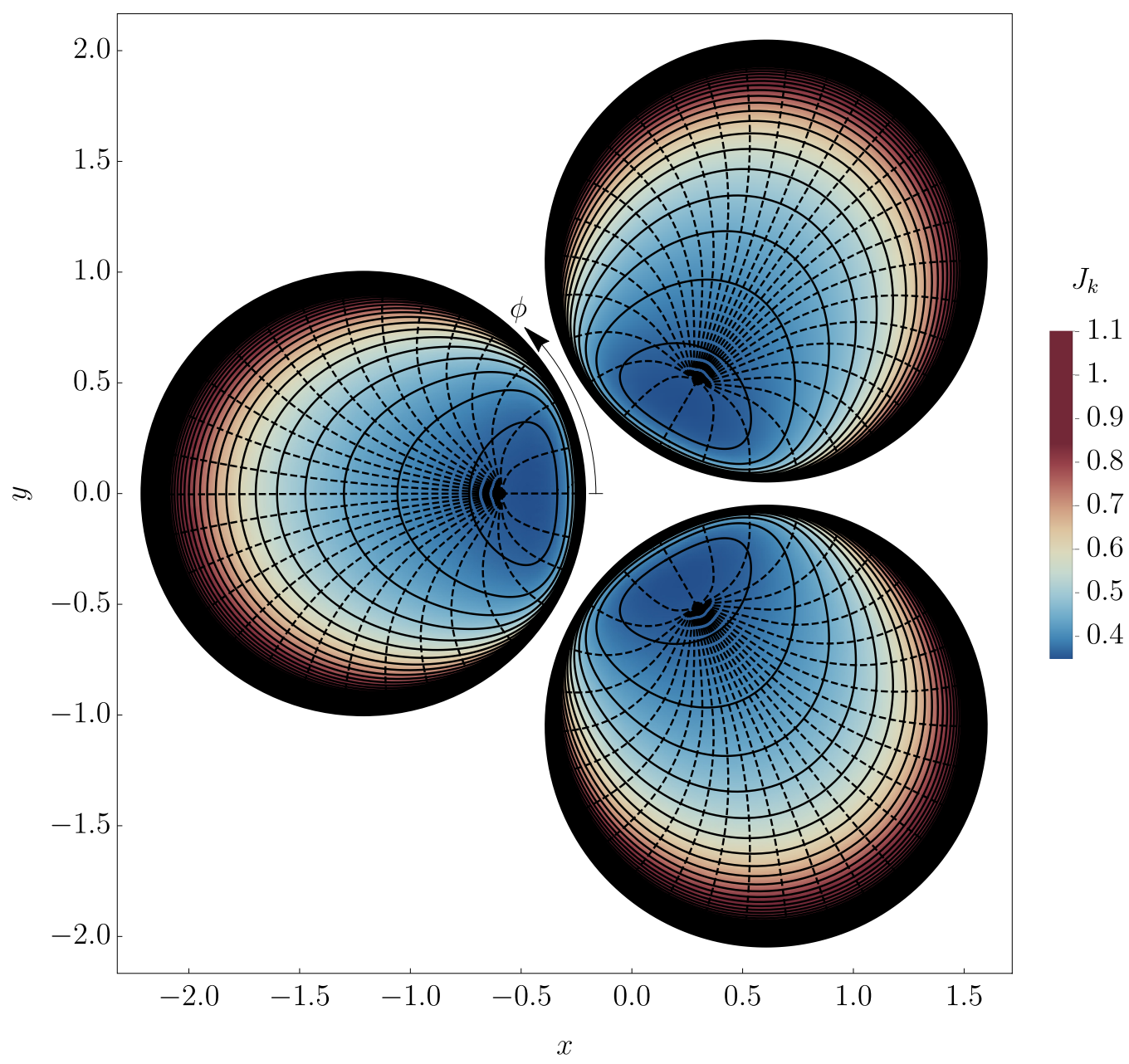

FIG. 6. Contours of the local evaporative fluxes $J_{k}$ for $k=1,2,3$ (solid curves) and the resulting streamlines of the depth-averaged flows (dashed curves) for a triplet of identical droplets of unit radius with their centers a distance $b \sin (\pi / 3)=2.1$ apart.

but it does not tell us anything directly about the accuracy of the theoretical predictions for the spatial distribution of the local evaporative flux or the resulting fluid flow within the droplet. For an isolated axisymmetric droplet, the fluid flow within the droplet is axisymmetric and the density of the deposit depends only on the initial distribution of particles and so, in particular, the density of the deposit does not depend on the details of the fluid flow. However, in nonaxisymmetric situations, such as the nonaxisymmetric droplets considered by Sáenz et al. [31] and the nonaxisymmetric evaporation of multiple droplets considered in the present work, understanding the details of the fluid flow is essential to determining the density of the deposit. Thus the comparison with experimental results described in the present work is perhaps the most stringent test of the diffusion-limited model to date, a test which it evidently passes remarkably well.

Finally, we note that the approach described in the present work is rather general and can, in principle, be applied to any arrangement of any number of thin droplets with pinned or unpinned contact lines. 


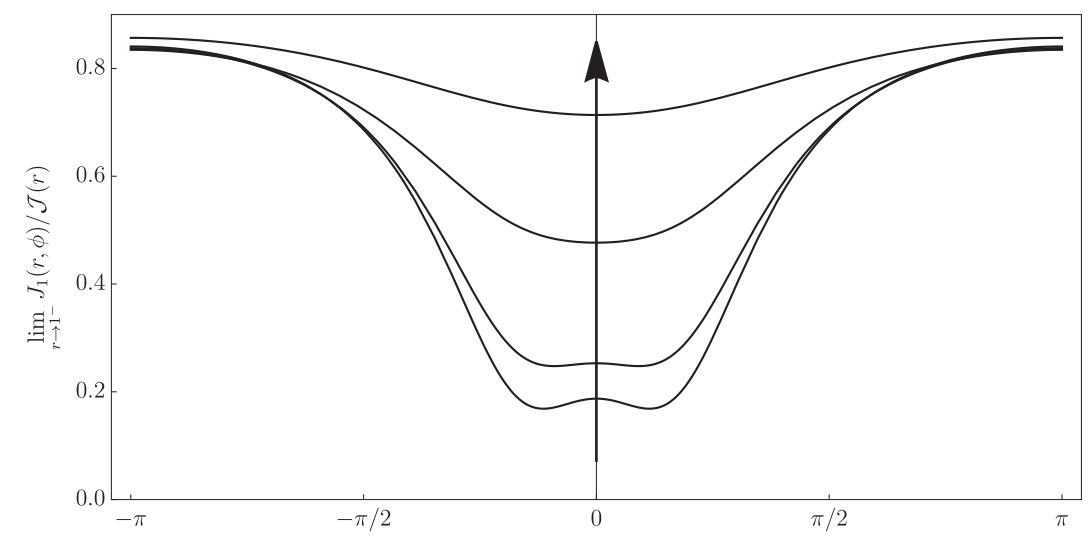

(a)

$\phi$

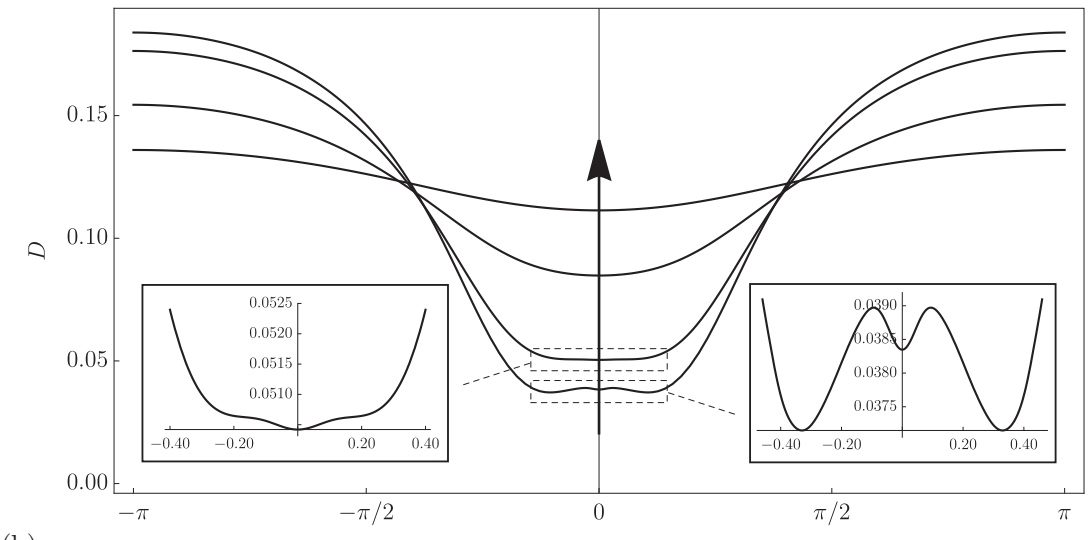

(b)

FIG. 7. (a) The normalized evaporative flux at the contact line, $\lim _{r \rightarrow 1^{-}} J_{1}(r, \phi) / \mathcal{J}(r)$, and (b) the density of the deposit $D$ as functions of the azimuthal coordinate $\phi$ for a triplet of identical droplets of unit radius with their centers distances $b \sin (\pi / 3)=2.1,2.25,3$, and 5 apart. In both parts the arrow indicates the direction of increasing $b$. The insets show enlargements of $D$ near $\phi=0$ when $b \sin (\pi / 3)=2.1$ and 2.25.

\section{ACKNOWLEDGMENTS}

The authors gratefully acknowledge valuable discussions with Hannah-May D'Ambrosio (University of Strathclyde) and Prof. Khellil Sefiane (University of Edinburgh) about various aspects of droplet evaporation.

\section{APPENDIX: SOLUTION IN THE LIMIT OF LARGE BOND NUMBER}

As mentioned at the end of Sec. I, the present analysis is for the most commonly studied case of small droplets in which capillary effects dominate over gravitational effects, corresponding to the limit of small Bond number. In this Appendix we describe the corresponding analysis in the case of large droplets, for which gravitational effects dominate over capillary effects, corresponding to the limit of large Bond number, in which even greater analytical progress is possible.

In the limit of large Bond number, the free surface of the droplet is flat, i.e., $h_{k}=h_{k}(t)$, except in a narrow region near the contact line which we may neglect [52], and so the volume of the (nearly cylindrical) droplet is now given by $V_{k}=\pi a_{k}^{2} h_{k}$. The local fluid fluxes are again given by Eq. (15), 
while the kinematic condition given by Eq. (21) simplifies to

$$
\frac{d h_{k}}{d t}-\frac{h_{k}^{3}}{3} \nabla^{2} p_{k}=-J_{k}
$$

and hence Eq. (23) becomes

$$
\frac{d h_{k}}{d t}=-\frac{1}{\pi a_{k}^{2}} \int_{\phi=0}^{\phi=2 \pi} \int_{r=0}^{r=a_{k}} r J_{k} d r d \phi
$$

and so the partial differential equation for $p_{k}$ given by Eq. (24) becomes

$$
\nabla^{2} p_{k}=\frac{3}{h_{k}^{3}}\left[J_{k}-\frac{1}{\pi a_{k}^{2}} \int_{\phi=0}^{\phi=2 \pi} \int_{r=0}^{r=a_{k}} r J_{k} d r d \phi\right] .
$$

In principle, the same approach as that used in the main body of the present work can be used to solve the corresponding problem for large droplets. However, for brevity, in this Appendix we simply show how to obtain explicit asymptotic expressions for the pressure, and hence for the fluid fluxes and the density of the deposit, for a well-separated pair of identical droplets.

Adopting the same notation as in Sec. IV, and, without loss of generality, taking $a_{1}=a_{2}=1$ and $h_{1}(0)=h_{2}(0)=1$, the evaporative flux from the left-hand droplet is again given by Eq. (45), which can be expanded as

$$
J_{1}=\frac{2}{\pi \sqrt{1-r^{2}}}\left[1-\frac{2}{\pi b}+\frac{4}{\pi^{2} b^{2}}(1-\pi r \cos \phi)\right]+O\left(b^{-3}\right)
$$

in the limit of well-separated droplets, $b \rightarrow \infty$. Hence, the equation for the pressure in the left-hand droplet, obtained by setting $k=1$ in Eq. (A3), can be expanded as

$$
\nabla^{2} p_{1}=\frac{6}{\pi h^{3}}\left[\frac{1}{\sqrt{1-r^{2}}}\left(1-\frac{2}{\pi b}+\frac{4}{\pi^{2} b^{2}}(1-\pi r \cos \phi)\right)-2\left(1-\frac{2}{\pi b}+\frac{4}{\pi^{2} b^{2}}\right)\right]+O\left(b^{-3}\right),
$$

with solution

$$
\begin{aligned}
\frac{\pi h^{3}}{3} p_{1} & =\left(1-\frac{2}{\pi b}+\frac{4}{\pi^{2} b^{2}}\right)\left[2 \log \left(1+\sqrt{1-r^{2}}\right)-r^{2}-2 \sqrt{1-r^{2}}\right] \\
& +\frac{8}{3 \pi r b^{2}}\left[1+r^{2}-\left(1-r^{2}\right)^{3 / 2}\right] \cos \phi+O\left(b^{-3}\right) .
\end{aligned}
$$

Hence, the local fluid fluxes can be expanded as

$$
\begin{aligned}
Q_{1}^{(r)} & =2\left(1-\frac{2}{\pi b}+\frac{4}{\pi^{2} b^{2}}\right) \frac{-1+r^{2}+\sqrt{1-r^{2}}}{\pi r} \\
& +\frac{8}{3 \pi^{2} r^{2} b^{2}}\left[1-r^{2}-\left(1+2 r^{2}\right) \sqrt{1-r^{2}}\right] \cos \phi+O\left(b^{-3}\right)
\end{aligned}
$$

and

$$
Q_{1}^{(\phi)}=\frac{8}{3 \pi^{2} r^{2} b^{2}}\left[1+r^{2}-\left(1-r^{2}\right)^{3 / 2}\right] \sin \phi+O\left(b^{-3}\right) .
$$

We now seek to determine the density of the deposit at a point on the contact line with polar angle $\phi=\phi_{\mathrm{C}}$, i.e., $D\left(\phi_{\mathrm{C}}\right)$, where $D$ is given by Eq. (50) with $M$ given by Eq. (49). Note that $M(2 \pi)=\pi$ for this cylindrical droplet. To do this we must first locate the source from which all of the streamlines emanate. By symmetry this must lie on the line of symmetry, $\phi=0$, and, by expanding $Q_{1}^{(r)}=0$ in powers of $b$, we find that it is located at $r=r_{\mathrm{S}}$ and $\phi=\phi_{\mathrm{S}}=0$, where

$$
r_{\mathrm{S}}=\frac{20}{3 \pi b^{2}}+O\left(b^{-3}\right)
$$


TABLE I. Comparison between the coefficients of the terms $b^{-2} \cos \phi_{\mathrm{C}}$ and $b^{-3} \cos \left(2 \phi_{\mathrm{C}}\right)$ obtained from the analytical prediction for $D$ given by Eq. (A14) and the values of $D$ obtained from Eqs. (49) and (50) for $b=3$ and $b=10$, respectively.

\begin{tabular}{lccc}
\hline \hline Term & Analytical Prediction Eq. (A14) & $b=3$ & $b=10$ \\
\hline$b^{-2} \cos \phi_{\mathrm{C}}$ & $-\frac{5 \pi+8}{6 \pi} \simeq-1.25775$ & -1.28 & -1.26 \\
$b^{-3} \cos \left(2 \phi_{\mathrm{C}}\right)$ & $-\frac{4(13+\log 4)}{15 \pi} \simeq-1.22115$ & -1.06 & -1.13 \\
\hline \hline
\end{tabular}

The streamline $\phi=\phi_{\mathrm{S}}\left(r ; \phi_{\mathrm{C}}\right)$ starting at the source at $r=r_{\mathrm{S}}$ and $\phi=\phi_{\mathrm{S}}=0$ and ending on the contact line at $r=1$ and $\phi=\phi_{\mathrm{S}}=\phi_{\mathrm{C}}$ satisfies

$$
\frac{d \phi_{\mathrm{S}}}{d r}=\frac{1}{r} \frac{Q^{(\phi)}}{Q^{(r)}}=\frac{4\left(3-r^{2}+2 \sqrt{1-r^{2}}\right)}{3 \pi r^{2} \sqrt{1-r^{2}} b^{2}} \sin \phi_{\mathrm{S}}+O\left(b^{-3}\right),
$$

which may be solved by expanding $\phi_{\mathrm{S}}$ in powers of $b$ to obtain

$$
\phi_{\mathrm{S}}\left(r ; \phi_{\mathrm{C}}\right)=\phi_{\mathrm{C}}+\frac{4\left[r(2+\arccos r)-2-3 \sqrt{1-r^{2}}\right]}{3 \pi r b^{2}} \sin \phi_{\mathrm{C}}+O\left(b^{-3}\right) .
$$

The density of the deposit at $\phi=\phi_{\mathrm{C}}$ is then given by

$$
D\left(\phi_{\mathrm{C}}\right)=\frac{d M}{d \phi_{\mathrm{C}}}=\frac{d}{d \phi_{\mathrm{C}}} \int_{r=r_{\mathrm{S}}}^{r=1} \int_{\phi=0}^{\phi=\phi_{\mathrm{S}}\left(r ; \phi_{\mathrm{C}}\right)} r d \phi d r,
$$

and hence

$$
D\left(\phi_{\mathrm{C}}\right)=\int_{r_{\mathrm{S}}}^{1} r \frac{\partial \phi_{S}}{\partial \phi_{C}} d r=\frac{1}{2}-\frac{5 \pi+8}{6 \pi b^{2}} \cos \phi_{\mathrm{C}}+O\left(b^{-3}\right) .
$$

The expansion given by Eq. (A13) can be continued to next order to obtain

$$
D\left(\phi_{\mathrm{C}}\right)=\frac{1}{2}-\frac{5 \pi+8}{6 \pi b^{2}} \cos \phi_{\mathrm{C}}-\frac{4(13+\log 4)}{15 \pi b^{3}} \cos \left(2 \phi_{\mathrm{C}}\right)+O\left(b^{-4}\right) .
$$

Higher-order corrections to $D\left(\phi_{\mathrm{C}}\right)$ may also be obtained, but rapidly become more complicated. Table I shows a comparison between the coefficients of the terms $b^{-2} \cos \phi_{\mathrm{C}}$ and $b^{-3} \cos \left(2 \phi_{\mathrm{C}}\right)$ obtained from the analytical prediction for $D$ given by Eq. (A14) and the values of $D$ obtained from Eqs. (49) and (50) for $b=3$ and $b=10$. The diminishing discrepancies as $b$ increases evident in Table I are due to the omitted higher-order corrections. In particular, analysis of these corrections indicates that the expression for $b^{-2} \cos \phi_{\mathrm{C}}$ has relative error $O\left(b^{-2}\right)$, while the expression for $b^{-3} \cos \left(2 \phi_{\mathrm{C}}\right)$ has relative error $O\left(b^{-1}\right)$, explaining the superior performance of the former.

[1] A. F. Routh, Drying of thin colloidal films, Rep. Prog. Phys. 76, 046603 (2013).

[2] R. G. Larson, Transport and deposition patterns in drying sessile droplets, AIChE J. 60, 1538 (2014).

[3] J. M. Stauber, S. K. Wilson, B. R. Duffy, and K. Sefiane, On the lifetimes of evaporating droplets, J. Fluid Mech. 744, R2 (2014).

[4] D. Brutin and V. Starov, Recent advances in droplet wetting and evaporation, Chem. Soc. Rev. 47, 558 (2018).

[5] F. Giorgiutti-Dauphiné and L. Pauchard, Drying drops, Eur. Phys. J. E: Soft Matter Biol. 41, 32 (2018).

[6] A. S. Dimitrov, C. D. Dushkin, H. Yoshimura, and K. Nagayama, Observations of latex particle twodimensional-crystal nucleation in wetting films on mercury, glass, and mica, Langmuir 10, 432 (1994). 
[7] J. Boneberg, F. Burmeister, C. Schäfle, P. Leiderer, D. Reim, A. Fery, and S. Herminghaus, The formation of nano-dot and nano-ring structures in colloidal monolayer lithography, Langmuir 13, 7080 (1997).

[8] M. Bale, J. C. Carter, C. J. Creighton, H. J. Gregory, P. H. Lyon, P. Ng, L. Webb, and A. Wehrum, Ink-jet printing: The route to production of full-color P-OLED displays, J. Soc. Inf. Disp. 14, 453 (2006).

[9] E. C. Tredenick, W. A. Forster, R. Pethiyagoda, R. M. van Leeuwen, and S. W. McCue, Evaporating droplets on inclined plant leaves and synthetic surfaces: Experiments and mathematical models, J. Coll. Int. Sci. 592, 329 (2021).

[10] R. D. Deegan, O. Bakajin, T. F. Dupont, G. Huber, S. R. Nagel, and T. A. Witten, Capillary flow as the cause of ring stains from dried liquid drops, Nature 389, 827 (1997).

[11] R. D. Deegan, Pattern formation in drying drops, Phys. Rev. E 61, 475 (2000).

[12] R. D. Deegan, O. Bakajin, T. F. Dupont, G. Huber, S. R. Nagel, and T. A. Witten, Contact-line deposits in an evaporating drop, Phys. Rev. E 62, 756 (2000).

[13] Y. O. Popov, Evaporative deposition patterns: Spatial dimensions of the deposit, Phys. Rev. E 71, 036313 (2005).

[14] R. Zheng, A study of the evaporative deposition process: Pipes and truncated transport dynamics, Euro. Phys. J. E 29, 205 (2009).

[15] A. Askounis, D. Orejon, V. Koutsos, K. Sefiane, and M. E. R. Shanahan, Nanoparticle deposits near the contact line of pinned volatile droplets: Size and shape revealed by atomic force microscopy, Soft Matter 7, $4152(2011)$.

[16] Y. Hamamoto, J. R. E. Christy, and K. Sefiane, Order-of-magnitude increase in flow velocity driven by mass conservation during the evaporation of sessile drops, Phys. Rev. E 83, 051602 (2011).

[17] Á. G. Marín, H. Gelderblom, D. Lohse, and J. H. Snoeijer, Order-to-disorder Transition in Ring-Shaped Colloidal Stains, Phys. Rev. Lett. 107, 085502 (2011).

[18] Á. G. Marín, H. Gelderblom, D. Lohse, and J. H. Snoeijer, Rush-hour in evaporating coffee drops, Phys. Fluids 23, 091111 (2011).

[19] P. J. Yunker, T. Still, M. A. Lohr, and A. G. Yodh, Suppression of the coffee-ring effect by shapedependent capillary interactions, Nature 476, 308 (2011).

[20] G. Berteloot, A. Hoang, A. Daerr, P. H. Kavehpour, F. Lequeux, and L. Limat, Evaporation of a sessile droplet: Inside the coffee stain, J. Coll. Int. Sci. 370, 155 (2012).

[21] A. Askounis, K. Sefiane, V. Koutsos, and M. E. R. Shanahan, Structural transitions in a ring stain created at the contact line of evaporating nanosuspension sessile drops, Phys. Rev. E 87, 012301 (2013).

[22] A. W. Wray, D. T. Papageorgiou, R. V. Craster, K. Sefiane, and O. K. Matar, Electrostatic suppression of the "coffee stain effect," Langmuir 30, 5849 (2014).

[23] F. Boulogne, F. Ingremeau, and H. A. Stone, Coffee-stain growth dynamics on dry and wet surfaces, J. Phys. Condens. Matter 29, 074001 (2016).

[24] S. Jafari Kang, V. Vandadi, J. D. Felske, and H. Masoud, Alternative mechanism for coffee-ring deposition based on active role of free surface, Phys. Rev. E 94, 063104 (2016).

[25] J. Y. Kim and B. M. Weon, Evaporation of strong coffee drops, Appl. Phys. Lett. 113, 183704 (2018).

[26] D. Mampallil and H. B. Eral, A review on suppression and utilization of the coffee-ring effect, Adv. Coll. Int. Sci. 252, 38 (2018).

[27] X. Yang, Z. Jiang, P. Lyu, Z. Ding, and X. Man, Deposition pattern of drying droplets, Commun. Theor. Phys. 73, 047601 (2021).

[28] W. Cheng, N. Park, M. T. Walter, M. R. Hartman, and D. Luo, Nanopatterning self-assembled nanoparticle superlattices by moulding microdroplets, Nat. Nanotechnol. 3, 682 (2008).

[29] X. Du and R. D. Deegan, Ring formation on an inclined surface, J. Fluid Mech. 775, R3 (2015).

[30] J. Y. Kim, I. G. Hwang, and B. M. Weon, Evaporation of inclined water droplets, Sci. Rep. 7, 42848 (2017).

[31] P. J. Sáenz, A. W. Wray, Z. Che, O. K. Matar, P. Valluri, J. Kim, and K. Sefiane, Dynamics and universal scaling law in geometrically-controlled sessile drop evaporation, Nat. Commun. 8, 14783 (2017).

[32] M. L. Timm, E. Dehdashti, A. J. Darban, and H. Masoud, Evaporation of a sessile droplet on a slope, Sci. Rep. 9, 19803 (2019). 
[33] I. I. Argatov, Electrical contact resistance, thermal contact conductance and elastic incremental stiffness for a cluster of microcontacts: Asymptotic modelling, Q. J. Mech. Appl. Math. 64, 1 (2011).

[34] B. Dollet and D. Lohse, Pinning stabilizes neighboring surface nanobubbles against Ostwald ripening, Langmuir 32, 11335 (2016).

[35] A. M. Lacasta, I. M. Sokolov, J. M. Sancho, and F. Sagués, Competitive evaporation in arrays of droplets, Phys. Rev. E 57, 6198 (1998).

[36] C. Schäfle, C. Bechinger, B. Rinn, C. David, and P. Leiderer, Cooperative Evaporation in Ordered Arrays of Volatile Droplets, Phys. Rev. Lett. 83, 5302 (1999).

[37] T. Kokalj, H. Cho, M. Jenko, and L. P. Lee, Biologically inspired porous cooling membrane using arrayeddroplets evaporation, Appl. Phys. Lett. 96, 163703 (2010).

[38] M. Sokuler, G. K. Auernhammer, C. J. Liu, E. Bonaccurso, and H.-J. Butt, Dynamics of condensation and evaporation: Effect of inter-drop spacing, Europhys. Lett. 89, 36004 (2010).

[39] T. K. Pradhan and P. K. Panigrahi, Deposition pattern of interacting droplets, Colloids Surf. A Physicochem. Eng. Asp. 482, 562 (2015).

[40] O. Carrier, N. Shahidzadeh-Bonn, R. Zargar, M. Aytouna, M. Habibi, J. Eggers, and D. Bonn, Evaporation of water: Evaporation rate and collective effects, J. Fluid Mech. 798, 774 (2016).

[41] G. Castanet, L. Perrin, O. Caballina, and F. Lemoine, Evaporation of closely-spaced interacting droplets arranged in a single row, Int. J. Heat Mass Trans. 93, 788 (2016).

[42] A. J. D. Shaikeea and S. Basu, Evaporating sessile droplet pair: Insights into contact line motion, flow transitions and emergence of universal vaporisation pattern, Appl. Phys. Lett. 108, 244102 (2016).

[43] A. Shaikeea, S. Basu, S. Hatte, and L. Bansal, Insights into vapor-mediated interactions in a nanocolloidal droplet system: Evaporation dynamics and affects on self-assembly topologies on macro- to microscales, Langmuir 32, 10334 (2016).

[44] S. Hatte, K. Pandey, K. Pandey, S. Chakraborty, and S. Basu, Universal evaporation dynamics of ordered arrays of sessile droplets, J. Fluid Mech. 866, 61 (2019).

[45] D. Khilifi, W. Foudhil, K. Fahem, S. Harmand, and J. S. Ben, Study of the phenomenon of the interaction between sessile drops during evaporation, Therm. Sci. 23, 1105 (2019).

[46] K. Pandey, S. Hatte, K. Pandey, S. Chakraborty, and S. Basu, Cooperative evaporation in two-dimensional droplet arrays, Phys. Rev. E 101, 043101 (2020).

[47] F. G. H. Schofield, A. W. Wray, D. Pritchard, and S. K. Wilson, The shielding effect extends the lifetimes of two-dimensional sessile droplets, J. Eng. Math. 120, 89 (2020).

[48] A. W. Wray, B. R. Duffy, and S. K. Wilson, Competitive evaporation of multiple sessile droplets, J. Fluid Mech. 884, A45 (2020).

[49] V. I. Fabrikant, On the potential flow through membranes, Z. Angew. Math. Phys. 36, 616 (1985).

[50] D. F. Swinehart, The Beer-Lambert law, J. Chem. Educ. 39, 333 (1962).

[51] G. van Rossum, Python Tutorial (Version 1.2), Report CS-R9526 (Centrum voor Wiskunde en Informatica, Amsterdam, The Netherlands, 1995).

[52] S. W. Rienstra, The shape of a sessile drop for small and large surface tension, J. Eng. Math. 24, 193 (1990). 